\title{
Modeling of Localization Error in Wireless Sensor Network*
}

\author{
Jinwon $\mathrm{CHOI}^{\dagger}$, Jun-Sung KANG ${ }^{\dagger}$, Yong-Hwa KIM ${ }^{\dagger \dagger}$, Nonmembers, and Seong-Cheol KIM ${ }^{\dagger a)}$, Member $^{\text {a }}$
}

\begin{abstract}
SUMMARY This letter presents the variation of localization error to network parameters, the number of range estimation results from anchor nodes (ANs) and average distance between ANs in centralized Wireless Sensor Network (WSN). In sensor network, ANs estimate the relative range to Target Node (TN) using Time-Of-Arrival (TOA) information of Ultra WideBand (UWB) radio and a fusion center determines the final localization of TN based on estimation results reported. From simulation results, the variation of localization error, which is defined as the difference between localization result of TN and its actual location, is represented as the function of number of estimation results to average distance between ANs. The distribution of localization error is matched to the Rician distribution whose K-factor value is given by the proposed formula as well. Finally, the normalized error function for the efficient localization network design is characterized.

key words: Wireless Sensor Network (WSN), range estimation, node localization and localization error
\end{abstract}

\section{Introduction}

The sensor networks composed of low-cost simple architecture sensors are now being constructed for a wide variety of applications. For the efficient deployment of Location Based Service (LBS) using a WSN, the accurate localization of a target sensor is strongly required. However, in LBS systems such as securing system in mountains and water quality monitoring system, it is almost impossible to design the arrangement of thousands sensor nodes in a systematic way. In these systems, as distribution of ANs, which estimate the location of a TN and report the estimation results to central processor, is unmethodical and numerous messages about the location of a TN are concentrated on the central processor, it is very hard to judge the exact location of TN efficiently. To design the effective WSN with predictable localization performance, it is proceeded to model the localization error property. Though many existing works have investigated about the range estimation algorithm in single node [1]-[5], the variation of localization error with the number of reporting ANs in a central processor, which is usually called as a fusion center, has not been modeled yet.

In this letter, we characterize the variation of localization error with the number of range estimation results from ANs considering an average distance between ANs. From

\footnotetext{
Manuscript received October 2, 2008.

${ }^{\dagger}$ The authors are with INMC and SOEE of Seoul National University, Seoul, Korea.

${ }^{\dagger}$ The author is with KERI, Korea.

*This work is partly supported by Seoul R\& BD program (10544) and BK21 project.

a)E-mail: sckim@maxwell.snu.ac.kr DOI: $10.1587 /$ transcom.E92.B.628
}

the simulation results, not only average localization error but also statistical distributions of localization error are investigated. The virtual WSN is assumed that TOA information of UWB is obtained for the range estimation in an AN [2]. Defining range estimation in an AN, the Cramer-Rao Lower Bound (CRLB) [2], [6] is used with channel parameters from standard channel model for IEEE 802.15 4a [7]. In fusion center, the localization is performed using conventional Least Squares (LS) algorithm based on the range estimation results from ANs and localization error is defined as distance difference between the estimated location of TN and its actual location [3], [4]. To describe the variation of localization error by an additional estimation result, the normalized error function is defined and characterized also.

\section{Simulation Scenarios}

\subsection{Node Distribution}

Simulation scenario is illustrated in Fig. 1. In simulation, 20 ANs are spread in 2-D virtual space whose size is determined by the average distance between ANs, $E(r)$ which varies from $10 \mathrm{~m}$ to $60 \mathrm{~m}$. The $\mathrm{TN}$ is assumed that it is located in the center of virtual space and the closest AN to TN is denoted $\mathrm{AN}_{1}$ while the furthest one is $\mathrm{AN}_{20}$. $20 \mathrm{ANs}$ are randomly distributed for 300 different scenarios in each $E(r)$ case. In every ANs' combination, the relative range estimations to $\mathrm{TN}$ and localization in a fusion center are repeated in Monte-Carlo simulation 300 times. Each AN estimates relative distance to $\mathrm{TN}$ and reports estimation result in every localization. Once ANs have been spread, it is assumed that the fusion center recognized the exact location of all ANs and the perfect communication links between fusion center and ANs were established.

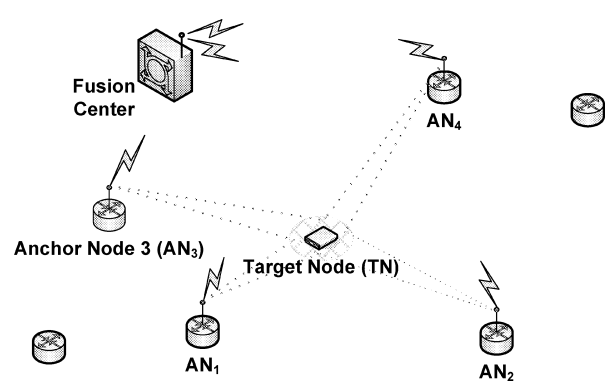

Fig. 1 Target Node (TN) and Anchor Nodes (ANs) in simulation. 


\subsection{Range Estimation in Anchor Node}

In this network, ANs estimate the relative distance to $\mathrm{TN}$ using the TOA information of UWB radio and report the estimation results to fusion center. Due to the additive noise and multi-path phenomenon, range estimations in ANs may have some extent of errors. When TOA of UWB radio is used for the range estimation, the accuracy of estimation with AWGN environment is represented as CRLB and it is given as [2], [6]

$$
\sqrt{\operatorname{Var}(\hat{d})} \geq \frac{c}{2 \sqrt{2} \pi \sqrt{S N R} \beta}
$$

where $c$ is speed of light, $S N R$ means the signal-to-noise ratio, and $\beta$ is the effective signal bandwidth. In simulation, received signal strength at each $\mathrm{AN}$ is calculated from transmitting power density, $-41.3 \mathrm{dBm} / \mathrm{MHz}$, and attenuation with the separation between $\mathrm{AN}$ and $\mathrm{TN}$ following the pathloss model of the proposed IEEE 802.15.4a outdoor NLOS channel model [7] while the noise floor level is computed as summation of the thermal noise and interference from other ANs. In attenuation, center frequency of $4.5 \mathrm{GHz}$ and $\beta$ of $500 \mathrm{MHz}$ are also considered for frequency dependent pathloss property. Moreover, the power delay profile, which shows the multipath model, is obtained from SalehValenzuela model with suggested parameters for detection of the first arrival path [7]. As noise of UWB communication link is assumed to follow Gaussian distribution in [7] and interference signals from other numerous ANs are assumed to be Gaussian distribution by the central limit theorem also, it is valid to assume that the distribution of range estimation $\tilde{d}_{i}$ at an $\mathrm{AN}_{i}$ follows Gaussian distribution. The mean of unbiased estimation is the actual distance $d_{i}$ while the standard deviation can be given from the lower bound of (1). Based on assumption that $\mathrm{AN}$ uses the efficient range estimators, mathematical expression of $\tilde{d}_{i}$ is given as follows:

$$
\tilde{d}_{i} \sim N\left(d_{i}, C R L B\right) .
$$

\subsection{Localization and Its Error in Fusion Center}

The localization of TN is carried out in a fusion center and graphically explained in Fig. 2. At first, the range circles of ANs, $C_{i}$ 's, are determined from the range estimation results and locations of ANs. The radius of $C_{i}$ is $\tilde{d}_{i}$ while center of range circle is the location of $\mathrm{AN}_{i}$. Using the $\mathrm{LS}$ algorithm, estimation of a TN location is obtained recursively on virtual space [3], [4]. This means that the location where minimizes the summation of square of distance to $C_{i}$ is determined as the final localization of TN, $L_{e s t}$. This is represented as

$$
L_{e s t}=\underset{L}{\arg \min }\left(\sum_{i}\left\|L-C_{i}\right\|^{2}\right)
$$

where $L$ means location on virtual space and $\|\cdot\|$ indicates

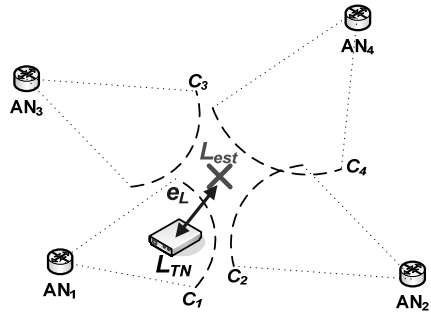

$C_{i}$ : Range circle from $\mathrm{AN}_{\mathrm{i}}(\mathrm{i}=1,2,3,4)$

$L_{T N}:$ Actual location of TN

$L_{e s t}:$ Estimated location of TN

$e_{L}$ : Localization Error, Distance difference between $L_{T N}$ and $L_{e s t}$

Fig. 2 Description of localization in fusion center with 4 ANs.

Euclidean distance. After $L_{e s t}$ is decided, the localization error, $e_{L}$, which is defined as the distance difference between $L_{e s t}$ and actual location of TN, $L_{T N}$ is given as

$$
e_{L}=\left\|L_{e s t}-L_{T N}\right\| .
$$

\section{Modeling of Localization Error}

\subsection{Localization Error Prediction Formula}

Localization error, $e_{L}$, varies to the number of range estimation results used for localization and $E(r)$. As an AN reports only one range estimation result in every localization, the number of range estimation results is denoted as $n_{A N}$. In selection of $n_{A N}$ at a fusion center, smaller $\tilde{d}_{i}$ values are firstly chosen. In Fig. 3, variation of $e_{L}$ to $n_{A N}$ is shown to each $E(r)$. These values are obtained from 90,000 localization results in every $E(r)$.

In every $E(r)$, gradual decrease of localization error is expressed as the linear function of natural logarithm of $n_{A N}$. The mathematical expression is given as

$$
e_{L}=a * \ln \left(n_{A N}\right)+b .
$$

Selected regression models are illustrated in Fig. 4. Conceptually, coefficient $a$ means the decline ratio of localization to $n_{A N}$ and coefficient $b$ is related to the initial localization error when $n_{A N}$ equals 3 in each $E(r)$. From the coefficient modeling, it is found that $a$ linearly increases while $b$ decreases with $E(r)$ as in Fig. 5. Both are represented as the linear functions of $E(r)$. Then, localization error prediction formula can be expressed from (5) using network parameters as

$e_{L}\left(E(r), n_{A N}\right)=(-0.03) * E(r) * \ln \left(n_{A N}\right)+0.19 * E(r)$.

The decline of $a$ to $E(r)$ means that additional range estimation results give relatively bigger impact on localization performance when $E(r)$ is bigger, the case of the ANs are more sparsely distributed. For $b$, raise with $E(r)$ indicates the increase of $e_{L}$ when number of available range estimation results is minimum, 3 . 


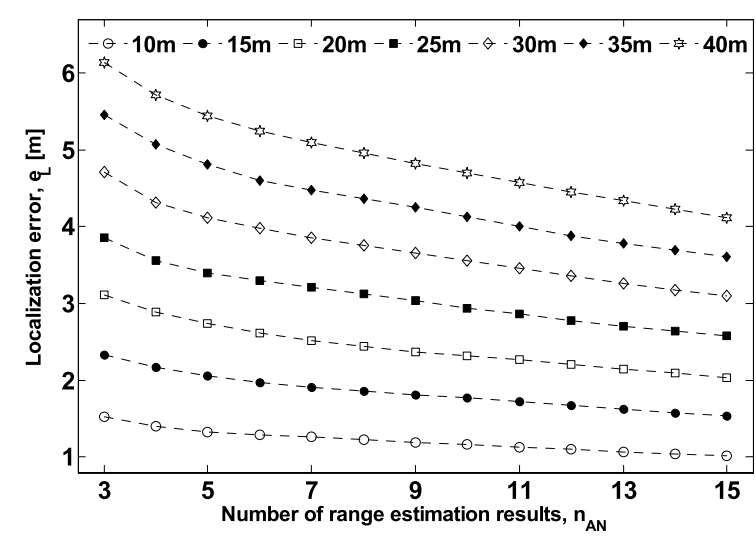

Fig. 3 Mean of localization error to $n_{A N}$ in each $E(r)$ value.

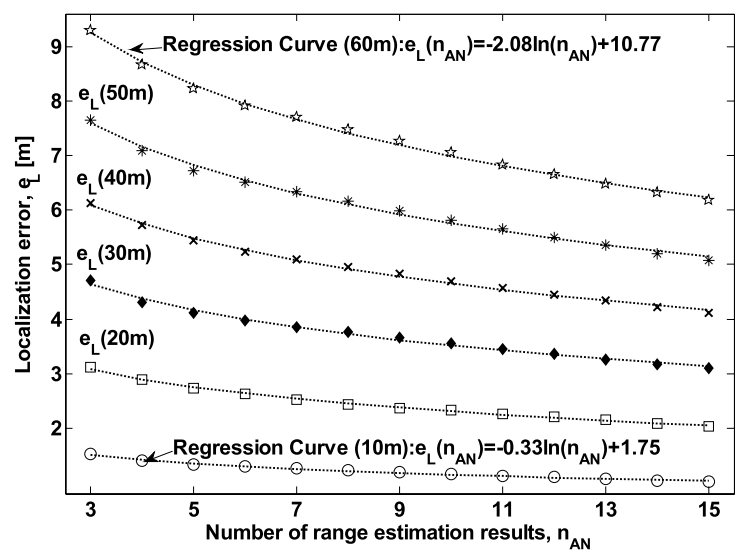

Fig. 4 Regression results of $e_{L}$ prediction formula with $n_{A N}$, in selected $E(r)$ values.

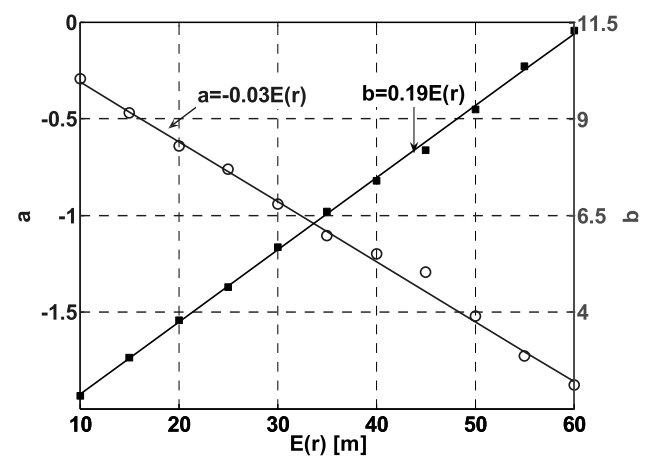

Fig. 5 Regression results about coefficients of $e_{L}$ function, $a$ and $b$.

\subsection{Distribution of Localization Error}

Even though the mean of $e_{L}$ is obtained from proposed error formula, the statistical distribution of localization error is also crucially needed for precise WSN performance design. Distribution of localization error in each $E(r)$ and $n_{A N}$ is compared to existing distributions (Gaussian, Rayleigh, and Rician distributions with various $\mathrm{K}$-factor values). To examine the closeness between $e_{L}$ distribution to existing ones,

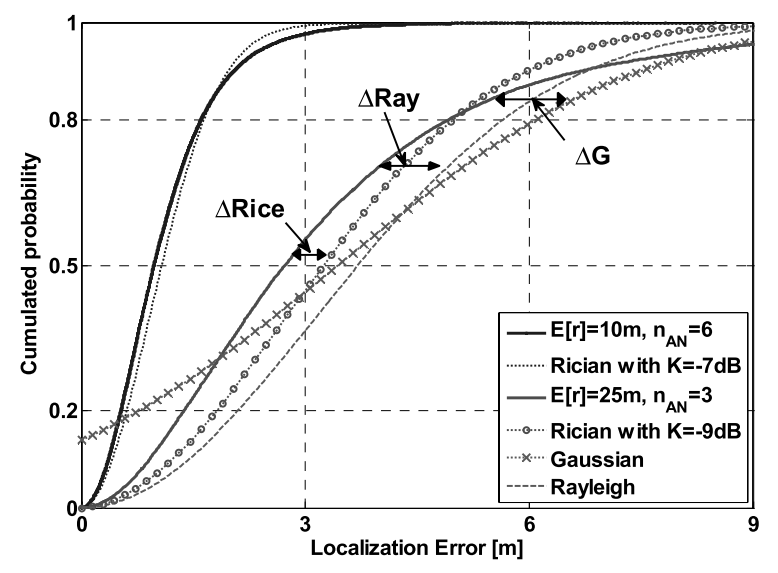

Fig. 6 Distribution regression results on simulation data of selected cases.

Table 1 Mean and standard deviations of normalized distribution gaps.

\begin{tabular}{|c||c|c|c|}
\hline$[\mathrm{dB}]$ & $\Delta \mathrm{G} / \mathrm{E}\left[\mathrm{e}_{\mathrm{L}}\right]$ & $\Delta \mathrm{Ray} / \mathrm{E}\left[\mathrm{e}_{\mathrm{L}}\right]$ & $\Delta \mathrm{Rice} / \mathrm{E}\left[\mathrm{e}_{\mathrm{L}}\right]$ \\
\hline \hline Mean & 0.114 & 0.075 & 0.047 \\
\hline STDEV & 0.051 & 0.056 & 0.032 \\
\hline
\end{tabular}

distribution gaps are defined as $\Delta \mathrm{G}, \Delta$ Ray, and $\Delta$ Rice for Gaussian distribution, Rayleigh one and Rician one, respectively. For Rician distribution, varying the K-factor of Rician distribution, the most similar Rician one to distribution of $e_{L}$ is selected. In Fig. 6, representative distributions of $e_{L}$, similar existing distributions and their distribution gaps are shown while the mean and standard deviation of normalized distribution gap values are summarized in Table 1. To compare the distribution gaps fairly, gap values are normalized to mean of $e_{L}$ in all $E(r)$ and $n_{A N}$ 's.

The distribution of localization error is most similar to the Rician distribution in all $E(r)$ and $n_{A N}$ 's. The mean distribution gap of Rician is about two thirds of that of Rayleigh and two fifths of that of Gaussians. For the closest Rician distributions, K-factors have small negative values in $\mathrm{dB}$ scale as $-7 \mathrm{~dB}$ when combination of network parameters, $\left(E(r), n_{A N}\right)$, is $(10,6)$ and $-9 \mathrm{~dB}$ when when $\left(E(r), n_{A N}\right)$ is $(25,3)$. K-factor of Rician distribution is represented as the function of network parameters also.

In Fig. 7, selected K-factor variation models to $n_{A N}$ of $E(r)$ values are plotted along with their formulations. In all $E(r)$ values, K-factor of each $E(r)$ increases gradually with $n_{A N}$. In same way, the $\mathrm{K}$-factor variation model with $E(r)$ in each $n_{A N}$ value is regressed as well. Consequently, based on these two K-factor variation models, the K-factor of Rician distribution describing $e_{L}$ is represented as the function $E(r)$ and $n_{A N}$ as given by

$$
\begin{aligned}
K\left(E(r), n_{A N}\right)= & 0.04 \cdot n_{A N} \cdot E(r)^{0.43} \\
& -0.05 \cdot E(r)-13[\mathrm{~dB}] .
\end{aligned}
$$




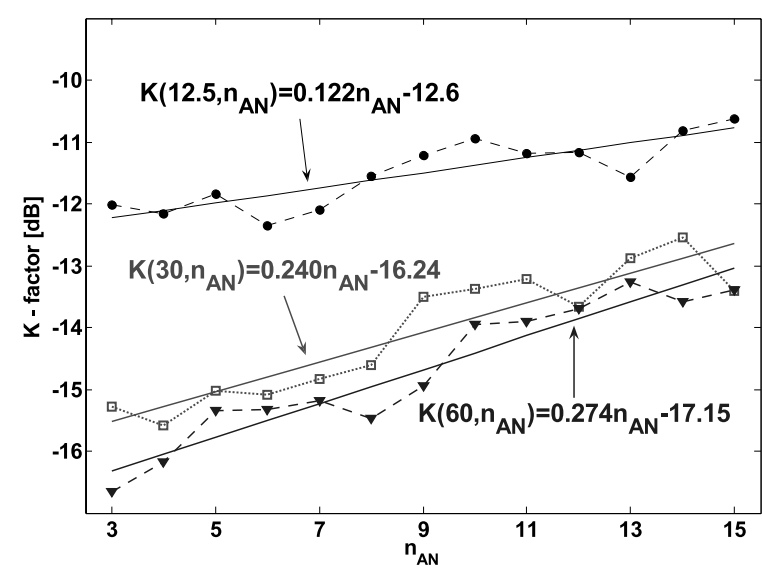

Fig. 7 Linear models of Rician K-factors to $n_{A N}$ in selected $E(r)$ 's.

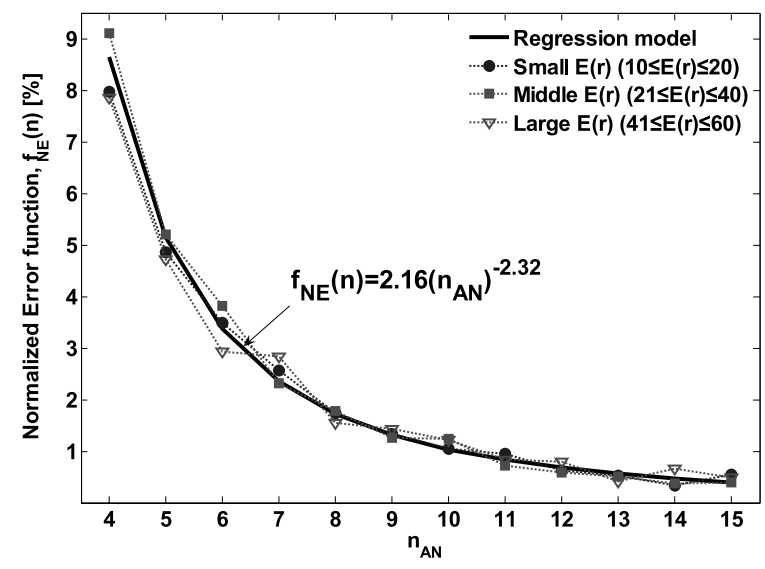

Fig. 8 Normalized error function to $n_{A N}$ and regression model.

\subsection{Normalized Error Function}

In general, it is obvious that more accurate localization can be performed as more range estimation results are used in localization. However, as a drawback, computational complexity is piled up to fusion center and this causes the degradation of localization speed. Using these practical localization error values, threshold for efficient localization algorithm is suggested.

To compare the decrease of localization error by additional estimation result, the normalized error function is defined as the function of $n_{A N}$ and given as

$$
f_{N E}\left(n_{A N}\right)=\frac{e_{L}\left(n_{A N}-1\right)-e_{L}\left(n_{A N}\right)}{e_{L}\left(n_{A N}-1\right)}\left(n_{A N}=4, \ldots 15\right) .
$$

This normalized error function indicates the relative amount of error reduction by one additional range estimation result.
Normalized error function to $n_{A N}$ is shown in Fig. 8 to three $E(r)$ groups. As shown in Fig. 8, the degradation ratio of localization error gets slowed regardless of $E(r)$. This degradation can be modeled as

$$
f_{N E}\left(n_{A N}\right)=2.16\left(n_{A N}\right)^{-2.32} \text {. }
$$

\section{Conclusion}

To predict the performance of practical location based services, the localization error prediction model should be set up in system design level. In this letter, the variation of localization error to network parameters, number of ANs and average distance between ANs, is modeled. The localization network are assumed that ANs estimate the relative distance to TN using TOA information of UWB radio and the fusion center determines the final localization of $\mathrm{TN}$ based on the LS algorithm. From simulation results, the localization error prediction formula is represented as the function of network parameters. In statistical characterization of localization error, it is found out that the distribution of localization error is regressed to Rician distribution with small K-factor value. This $\mathrm{K}$-factor can be obtained from suggested formula of network parameters as well. In final, the normalized error function describing the reduction of localization error by additional estimation result is established.

\section{References}

[1] N. Patwari, J.N. Ash, S. Kyperountas, A.O. Hero, III, R.L. Moses, and N.S. Correal, "Locating the nodes: Cooperative localization in wireless sensor networks,” IEEE Signal Mag., vol.22, no.4, pp.54-69, July 2005 .

[2] S. Gezici, Z. Tian, G.B. Giannakis, H. Kobayashi, A.F. Molisch, H.V. Poor, and Z. Sahinoglu, "Localization via ultra-wideband radios: A look at positioning aspects for future sensor networks," IEEE Signal Mag., vol.22, no.4, pp.70-84, July 2005.

[3] P.C. Chen, "A non-line-of-sight error mitigation algorithm in location estimation," Proc. IEEE WCNC, vol.1, pp.316-320, New Orleans, LA, Sept. 1999.

[4] S. Venkatesh and R.M. Buehrer, "A linear programming approach to NLOS error mitigation in sensor networks," Proc. IEEE Int. Conf. on IPSN, pp.301-308, Nashville, TN, April 2006.

[5] D.B. Jourdan, D. Dardari, and M.Z. Win, "Position error bound for UWB localization in dense cluttered environments," Proc. IEEE ICC, vol.8, pp.3705-3710, Istanbul, Turkey, June 2006.

[6] S.M. Kay, Fundamentals of statistical signal processing: Estimation Theory, pp.53-56, Prentice Hall PTR, 1993.

[7] A.F. Molisch, D. Cassioli, C.C. Chong, S. Emami, A. Fort, B. Kannan, J. Karedal, J. Kunisch, H.G. Schantz, K. Siwiak, and M.Z. Win, "A comprehensive standardized model for ultrawideband propagation channels," IEEE Trans. Antennas Propag., vol.54, no.11, pp.31513166, Nov. 2006 